\title{
IMAGE PROCESSING FOR CONSTRUCTION EQUIPMENT IDLE TIME ANALYSIS
}

\author{
Junhao Zou (jzou@ualberta.ca) \\ Grad. Research Assistant, University of Alberta, Edmonton, Alberta, T6G 2G7, Canada \\ Hyoungkwan Kim (hyoungkwan@ualberta.ca) \\ Assistant Professor, University of Alberta, Edmonton, Alberta, T6G 2G7, Canada
}

\begin{abstract}
Accurate analyses of equipment idle time is crucial for the efficient utilization of construction equipment in a large construction project. The less idle time the equipment has, the higher productivity the construction can achieve. However, it is not practical to measure construction equipment idle time by human visual observation. This paper describes an image processing-based methodology to automatically quantify the idle time of construction equipment. The image color space, HSV (Hues, Saturations, and Values), is used as the basis for image segmentation and tracing algorithms. The HSV color space shows significant advantages over the RGB (Red, Green, and Blue) color space in identifying and tracing construction equipment. The centroid coordinates of equipment are used as the indicators of movement. Experimental results show that the proposed methodology has a promising potential for effective equipment management in construction projects.
\end{abstract}

Keywords: Construction, Digital imaging, Equipment, Idle time.

\section{INTRODUCTION}

Along with labor and material, equipment is generally one of the three major resources required for large construction projects. The success of construction projects greatly depends on how appropriately construction equipment is planned for and allocated, and how efficiently it is utilized for a variety of construction tasks. One way to understand the level of efficiency of equipment utilization on a site is to measure the "idle time" of the equipment. Here, the idle time is defined as the amount of time during which the equipment is supposed to move on the job site to do the actual construction work, but is not moving. For example, even though a piece of equipment's engine is started, if the equipment is not significantly moving to do the allocated construction task, the equipment is not adding any value to the construction project. Therefore, the equipment is considered to be in idle time.

Minimizing the idle time of any deployed equipment has been of major interest in the construction industry. From the standpoint of the contractors who choose to rent equipment, the rental fee of equipment and the cost of related labor can be significantly reduced by minimizing the equipment idle time. From the perspective of the construction project, better planning and deployment of equipment based on thorough and accurate equipment idle time analyses will result in improved construction productivity, which can lead to significant time and cost savings. In any case, a good understanding of the equipment idle time is an essential prerequisite for developing a high-quality equipment deployment plan.

It is now not uncommon to see cameras installed on construction sites to monitor the construction activities. The cameras can be installed on top of an adjacent building, tower cranes, or some installation posts temporarily built just for the cameras. The cameras produce images of the construction field every 5 to 30 seconds; these can be sent to a computer in the management office or displayed on an Internet website, so that various project participants can understand what is going on in the construction field. These abundant image data of the construction site provide a unique opportunity to use an image processing-based approach to automatically measure construction equipment idle time.

There have been research efforts made to use the image processing technique for construction site monitoring. Neto and Arditi (2002) used color information in the RGB (Red, Green, and Blue) color space to identify a structural component (pier structure) on a bridge construction site [1]. Wu and Kim (2004) used a data fusion approach to detect and measure concrete columns on a general building construction site [2]. One important feature common to the two studies is that the objects of interest in the image data are stationary. For example, in the case of column construction, once the location of a particular column is identified, as long as the monitoring camera's angle and location is not changed, the same image region can be assumed to contain the same column's information. However, this assumption cannot be made when 
identifying mobile objects in image data. Mobile objects, such as construction equipment, change their location in image data over time by moving to other parts of the construction site. Moreover, since three-dimensional equipment is being reflected on the two-dimensional image space, the shapes that are projected in the two-dimensional image can also vary over time when the equipment changes its direction of movement or rotates. These characteristics of mobile object monitoring, especially in an outdoor environment like almost any construction site, present a big challenge to the effort to automatically analyze equipment idle time using image processing.

In this paper, we present an image processing-based methodology for analyzing the idle time of construction equipment. Image data obtained from the early phase of the construction of the Natural Resources Engineering Facility of the University of Alberta in Edmonton, Canada, are used to show the validity of the proposed methodology. During the foundation excavation activity, images of a hydraulic excavator operating on the construction site were taken every 10 seconds. By detecting the motion of the equipment on the consecutive images, an estimation of equipment idle time was achieved. There are three main technical issues to be considered in accurately estimating equipment idle time. The first is how to distinguish the hydraulic excavator from its complicated background region, including the land with natural soils, the land covered with snow, and other equipment. The second is how to trace the hydraulic excavator through all the images taken at 10 second time intervals. The last issue is how to use a certain indicator to determine the status of equipment movement, i.e., whether the equipment is idle or not. We describe the details of the proposed methodology in section 2 of this article. In section 3 , we evaluate the proposed methodology using 3 hours of image data (1080 images). Finally, section 4 will provides some discussion about future work and conclusions.

\section{DETAILED METHODOLOGY AND BODY OF WORK}

Before we describe the details of our methodology, we review some features and assumptions of this research as follows:

First, the resolution of the image data is $640 \times 480$ and the clarity is not very good. So we tried to perform as few image operations as possible to keep more accurate information about the hydraulic excavator. In addition, fewer operations for each image allow us to save a significant amount of processing time.
Second, since the interval between every two successive images is 10 seconds, we assume that the color range of the hydraulic excavator does not change significantly within these 10 seconds. Then, when we compare the same equipment in the consecutive images, the color information can be used to identify and trace the equipment movement.

Third, because of the nature of hydraulic excavators, we assume that its moving speed is not too fast and that there is no other equipment working in the range of its boom and bucket.

Finally, the assumption made for idle time estimation is that the hydraulic excavator is treated as working for 10 seconds, so long as the next image shows a significantly different position or orientation. This may cause some errors in the estimation results compared to the actual working time, which could be less than 10 seconds. However, the errors should be acceptable considering the short time interval (10 seconds) between two consecutive images.

\subsection{Target segmentation}

Target segmentation is the most challenging task, as is the case in many other image processing related researches. Fig.1, Fig.2, and Fig.3 show the color ranges (Red and Green, Red and Blue, Green and Blue, respectively) of the hydraulic excavator of interest and its background region. As can be seen from these figures, it is hard to extract the hydraulic excavator in the RGB (Red, Green, and Blue) color space by choosing a simple pattern classifier because some regions of the equipment have the same, or very similar, color ranges to those of the background region. Thus, the RGB color space is considered to be an inadequate feature space to distinguish the hydraulic excavator from the background region, which sometimes has snow and earth on the ground. This is somewhat in contrast to a previous research effort where only RGB color information is used to detect equipment [6].

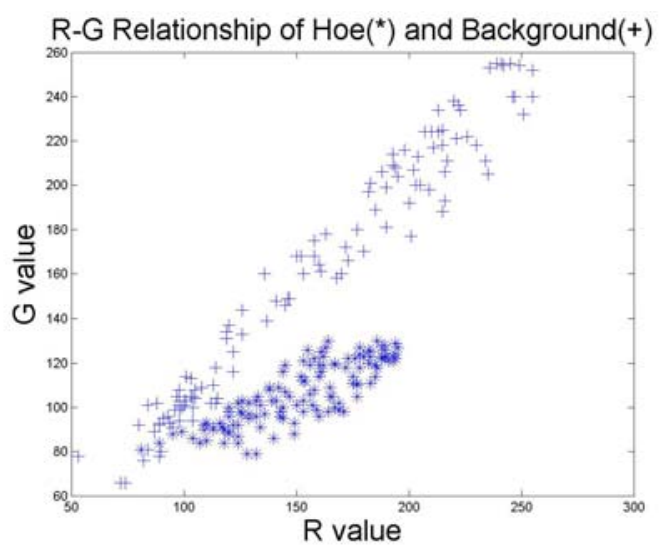

Fig.1 R-G Relationship of the Hydraulic Excavator(*) and its Background(+) 


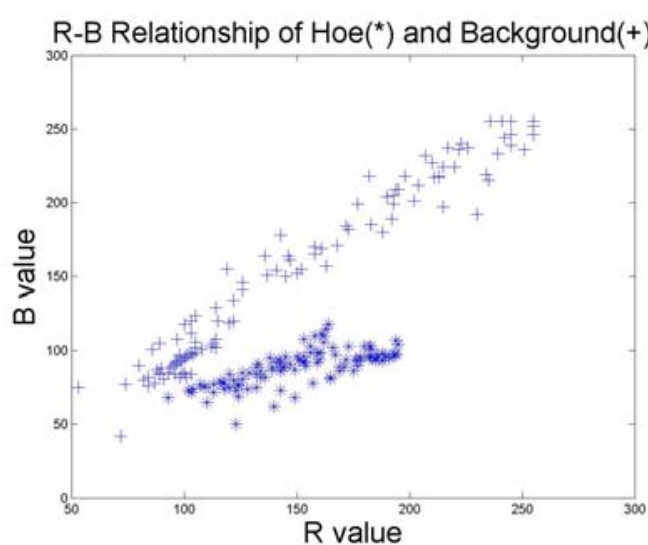

Fig.2 R-B Relationship of the Hydraulic Excavator(*) and its Background(+)

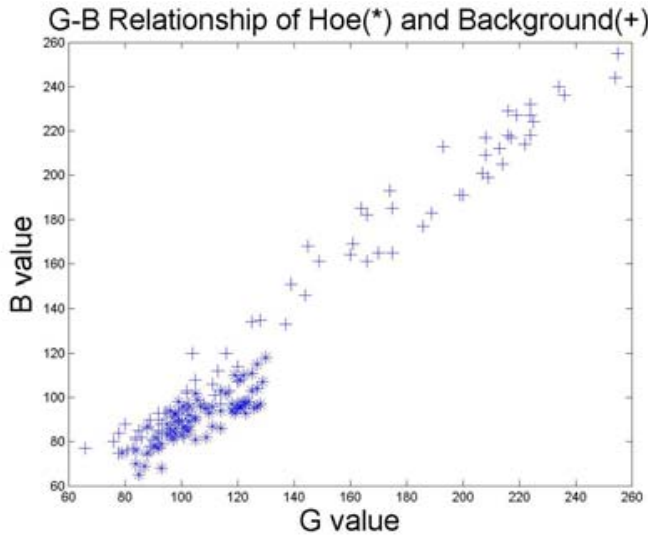

Fig.3 G-B Relationship of the Hydraulic Excavator(*) and its Background(+)

Fig. 4 and Fig. 5 are a sample original image and its grayscale version. As seen from Fig.5, it is almost impossible to extract the equipment from the grayscale image because the grayscale of the hydraulic excavator is very close to the grayscale of its background. Thus, adjusting the grayscale of images to highlight the hydraulic excavator is not considered to be a good approach.

Fig.6 illustrates the concept of the HSV color space. Hue determines the color; "As hue varies from 0 to 1.0, the corresponding colors vary from red, through yellow, green, cyan, blue, and magenta, back to red" [5]. Saturation means the level of shades of gray; “As saturation varies from 0 to 1.0, the corresponding colors (hues) vary from unsaturated (shades of gray) to fully saturated (no white component)" [5]. Value means the brightness; "As brightness varies from 0 to 1.0 , the corresponding colors become increasingly brighter" [5].

Since the main components of the background are black earth and white snow, their saturation values should be very close to zero. Fig.7 is the saturation image of the original image, and it certifies our inference. In the saturation image (Fig.7), most of the noise is removed and the hydraulic excavator is the most remarkable object. This indicates that saturation images greatly facilitate our target segmentation.

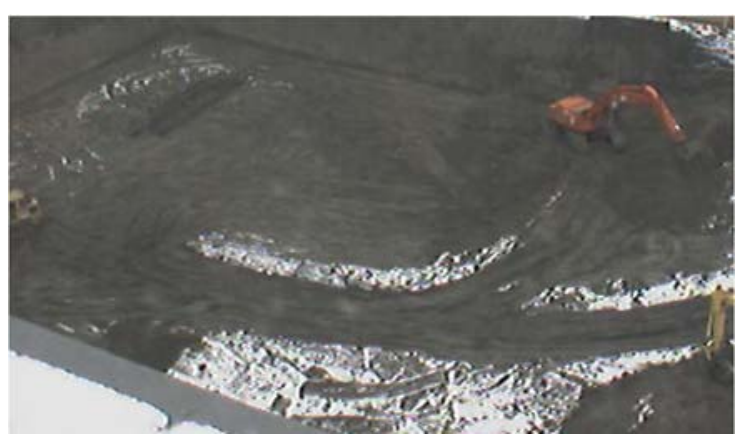

Fig.4 Original Image

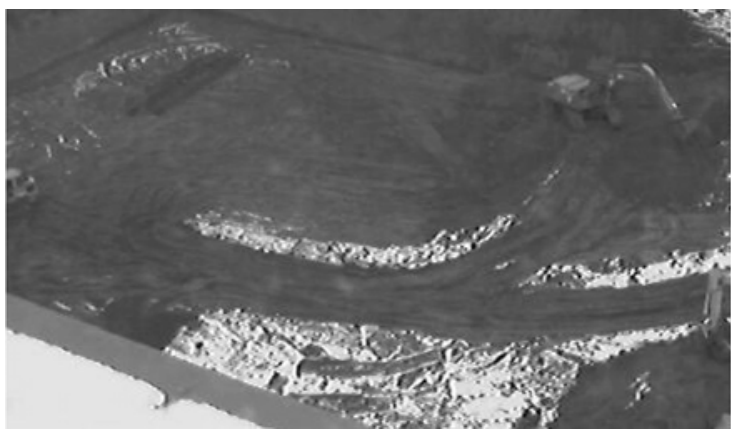

Fig.5 Grayscale Image of Original Image

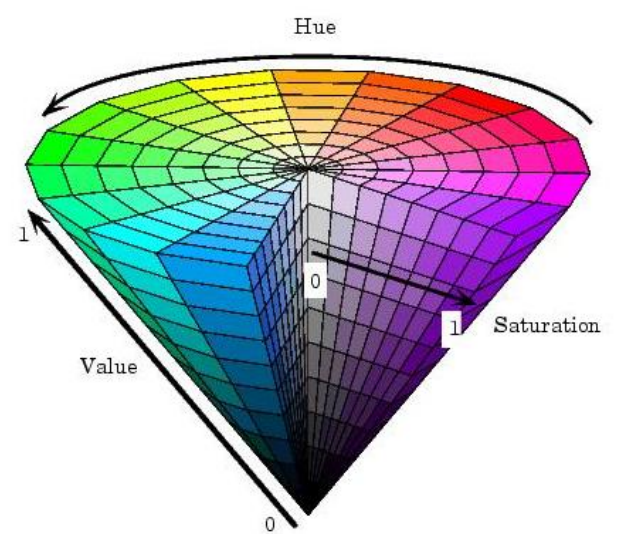

Fig.6 Concept of HSV [5]

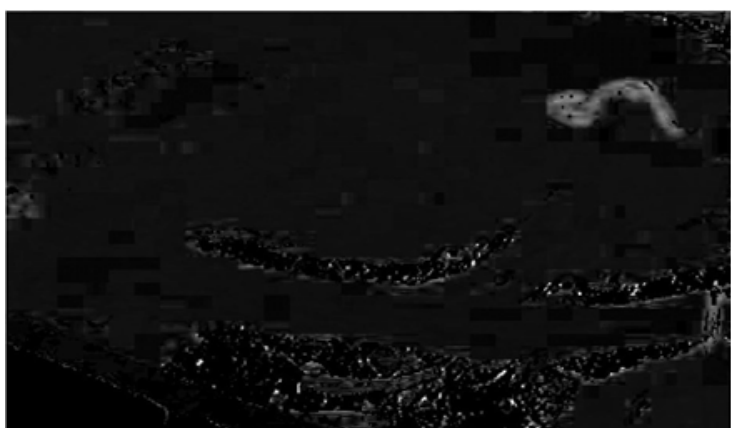

Fig.7 Saturation Image of Original Image

The application of other sophisticated algorithms, 
such as Canny Edge Detector and Watershed Transformation, to the saturation images did not achieve a satisfactory segmentation result unless the algorithms were combined with some pre or/and post image processing. These preliminary tests indicated that setting a threshold in the HSV color space is the easiest and simplest way to crop the hydraulic excavator. Fig.8, Fig.9, and Fig.10 show the segmentation results of the aforementioned three methods - Canny Edge Detector, Watershed Transformation, and threshold setting method, respectively.

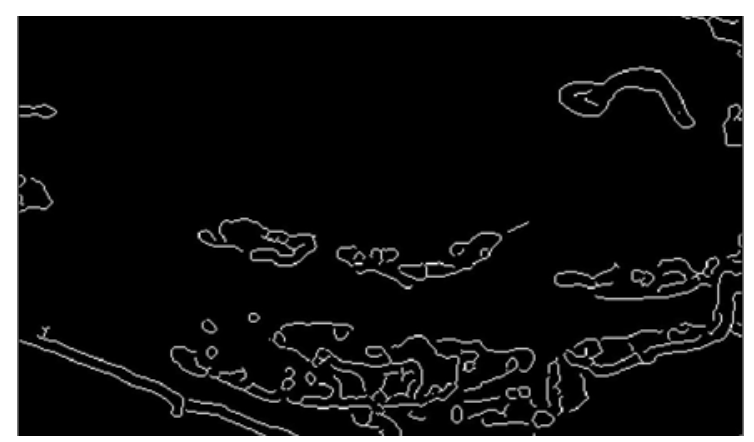

Fig.8 Result of Canny Edge Detector on the Saturation Image

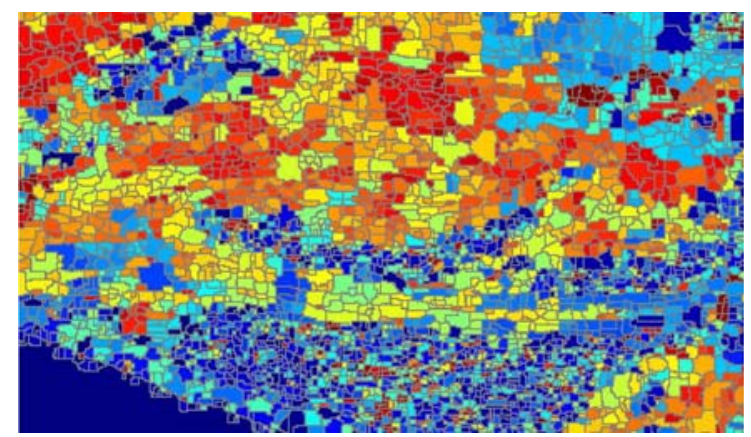

Fig.9 Result of Watershed Transformation on the Saturation Image

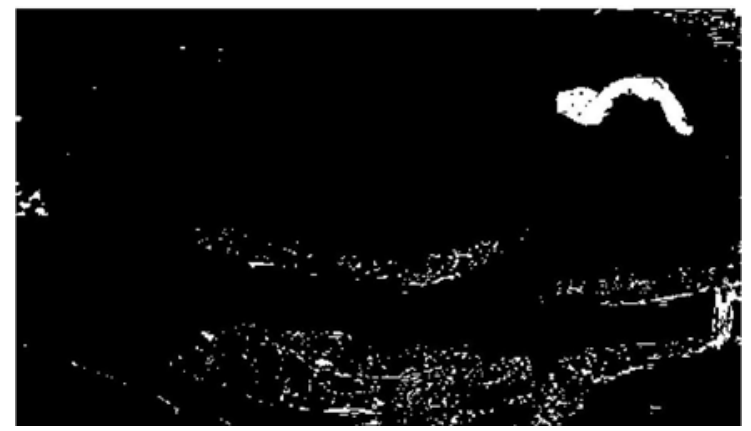

Fig.10 Result of Setting Thresholds on the Saturation Image

After removing some noise with fewer pixels and filling the holes in the object of interest, the image segmentation is finished. Fig.11 shows the final result.

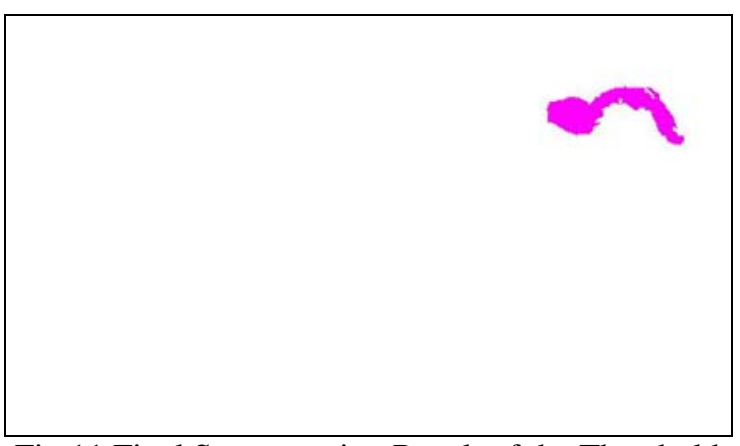

Fig.11 Final Segmentation Result of the Threshold Setting Approach in the HSV Space

\subsection{Object Tracking}

After image segmentation, how to trace the hydraulic excavator becomes the main problem. Generally, some features, such as the unique color range, shape features, and size features of the object of interest, are identified and used for this type of object tracking. Color range information, however, could not be used as an object identifying feature because other equipment with a similar color range can appear on the job site, thus causing confusion. The frequently changing shape of the hydraulic excavator on the image data also prevented us from relying on shape features, because shape feature extraction techniques, such as cross correlation function, would not work. The area number of pixels) of the hydraulic excavator also varies significantly when the equipment changes its direction of movement or appears on different parts of the site. Furthermore, some other hydraulic excavators of almost the same size appeared on the job site. Therefore, size could not be utilized to trace the hydraulic excavator in our research.

To address the aforementioned concerns, we used the concept of distance between objects. Once we identified the location of the hydraulic excavator in the current image, we assumed that the location of the equipment in the next image (10 seconds later) would be close to the location identified in the current image. Therefore, we identified the hydraulic excavator as the object in the current image with the closest centroid distance from the hydraulic excavator in the preceding image. In other words, the centroid of each object in the current image was calculated and the object with the centroid closest to that of the hydraulic excavator in the previous image was identified as the same piece of equipment. Although this approach generally showed good results, when two hydraulic excavators were positioned very close to one another, their identities were sometimes confused. To avoid these exceptional situations, image data based on shorter time intervals (such as 5 seconds), or on additional features of the equipment, could be used.

Other problems can arise, as illustrated in Fig.12, 
13, and 14. When the hydraulic excavator swings its boom in a certain direction, as shown in Fig.12, the steer room may divide the hydraulic excavator into two parts, as shown in Fig. 13.

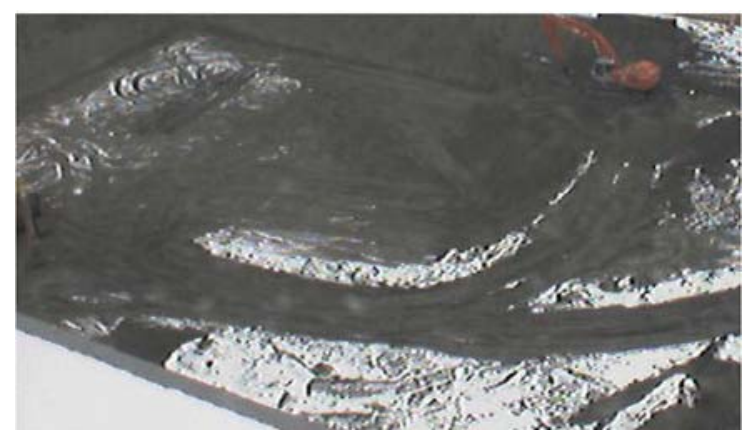

Fig.12 Hydraulic Excavator in a Certain Direction

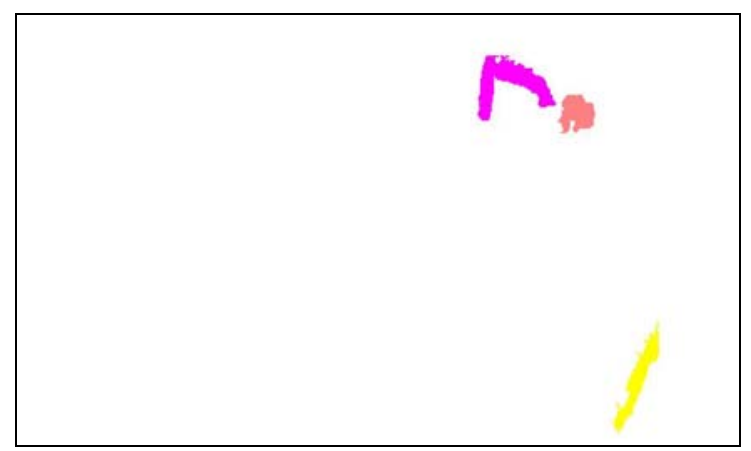

Fig.13 Segmentation Error

To remove this effect, which influences the final equipment idle time estimation, image dilation was performed when the program detected two segmented regions in very close proximity to one other. To reduce the deformation caused by image dilation, in this case by the shape of the hydraulic excavator, a $3 \times 3$ structuring element was used. Fig.14 shows the correct segmentation result after the dilation operation.

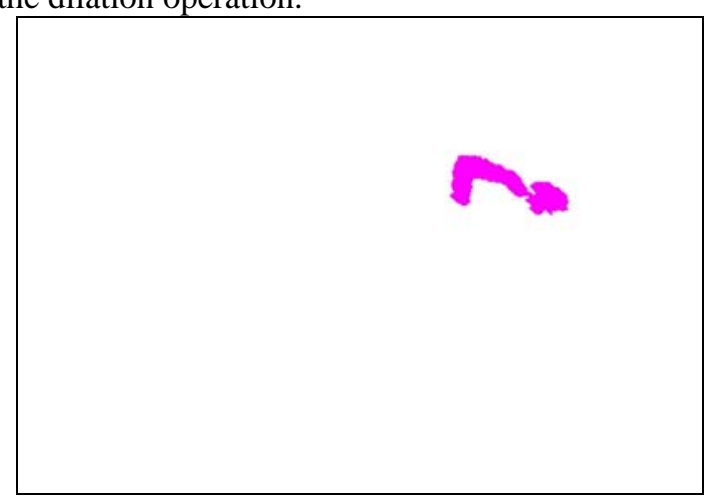

Fig.14 Image Dilation Result

\subsection{Movement Indicator}

Determining whether or not the equipment actually moves is an important step in measuring equipment idle time. For this purpose, many region properties, such as the coordinates of top-left, top-right, bottom-left, and bottom-right, and the length and orientation of major and minor axes could theoretically be used. However, in reality, these options are no so feasible. Any small effects caused by different lighting and weather conditions can hamper the quality of the images. Therefore, the centroid coordinates of the segmented region, as the simplest indicator of movement, were tried.

Selection of the movement indicator threshold value is another important factor that affects the accuracy of idle time estimation. Since perfectly stationary equipment can have different centroid coordinates in consecutive images, a certain threshold value in the form of a pixel distance must be chosen to determine whether or not the equipment moves. Thus, when the distance between the locations of an object's centroid differs by more than the threshold value between two consecutive images, then the object is considered to have moved. If this threshold value is too big, the image processing system will exaggerate equipment idle time; if it is too small, the system will underestimate the idle time. After trials of 300 images in three different time sections, a 1.6-pixel distance was chosen to be the criterion for equipment movement.

\subsection{Program Architecture}

A computer program was developed using MATLAB to automatically estimate the idle time of equipment. In addition to the aforementioned functions, two other functions were added to our program. One was resizing the original images to include only the areas relevant to our research. This allowed us to avoid unwanted interference from other objects in the image and significantly reduce processing time. The other was the object selection function which allows users to select any object of interest from the original image. Fig.15 shows the flow chart of the whole program.

\section{DISCUSSION OF RESULTS}

With the help of the proposed methodology, a high accuracy was achieved when a three-hour long image series was processed. The results show that the hydraulic excavator worked 7000 seconds during the 10800-second investigation period, with a working rate of $64.8 \%$ and an idle time of 3800 seconds. The real working rate by visual observation of the original images was $67.0 \%$. This gives a discrepancy of only $2.2 \%$. A further comparison of the results of each image processing shows that there are 44 images that are incorrectly analyzed out of a total of 1080 images. The error rate is $4.1 \%$. We think the accuracy of the results can meet the requirements of real project management.

A 1.6-pixel distance was chosen to be the 
movement indicator threshold value for determining the movement condition of the object of interest. But this value may not suit other research involving different types of equipment, camera resolutions, lighting conditions, etc. Furthermore, the location of the hydraulic excavator also affects the threshold value. For example, a hydraulic excavator located 100 meters away from the camera will certainly require a different movement indicator threshold value than will the same hydraulic excavator located just 50 meters away from the camera. Therefore, a careful calibration process should be conducted before carrying out the idle time analysis.

\section{CONCLUSIONS AND FUTURE WORK}

This paper presented the promising outcome of an image processing-based methodology which can help project managers or equipment managers better understand their equipment usage. Using the HSV (Hue, Saturation, and Value) color space as a platform for the simple threshold setting approach, the proposed methodology was able to effectively measure the idle time of a hydraulic excavator.

Currently, it takes about four seconds to process one image. Further improving image processing efficiency will be an important step towards bringing this research to fruition. Further tests of this methodology with more image data will also increase its reliability in dealing with different backgrounds and different types of equipment.

\section{REFERENCES}

[1] J. A. Neto and D. Arditi (2002). "Using Colors to Detect Structural Components in Digital Pictures", Computer-Aided Civil and Infrastructure Engineering, Vol. 17, No. 1, pp. 61-67.

[2] Y. Wu and H. Kim (2004). "Digital Imaging in Assessment of Construction Project Progress", Proc., 21st International Symposium on Automation and Robotics in Construction, IAARC, Jeju, Korea, Sep.

[3] H. Gao, W. Siu, and C. Hou (2001). "Improved Techniques for Automatic Image Segmentation”, IEEE Transactions on Circuits and Systems for Video Technology, Vol. 11, No. 12, pp.1273-1280.

[4] P. Soille (2000). "Morphological image analysis applied to crop field mapping”, Image and Vision Computing, Vol. 18, No. 13, pp. 1025-1032.

[5] The MathWorks, Image Processing Toolbox for Use with MATLAB*: User's Guide Version 4, 2003.
[6] ElGhandour, W. (2003). "Automated Real-Time Data Acquisition System", 10th Annual Canadian Construction Research Forum, Edmonton, Canada, Aug.

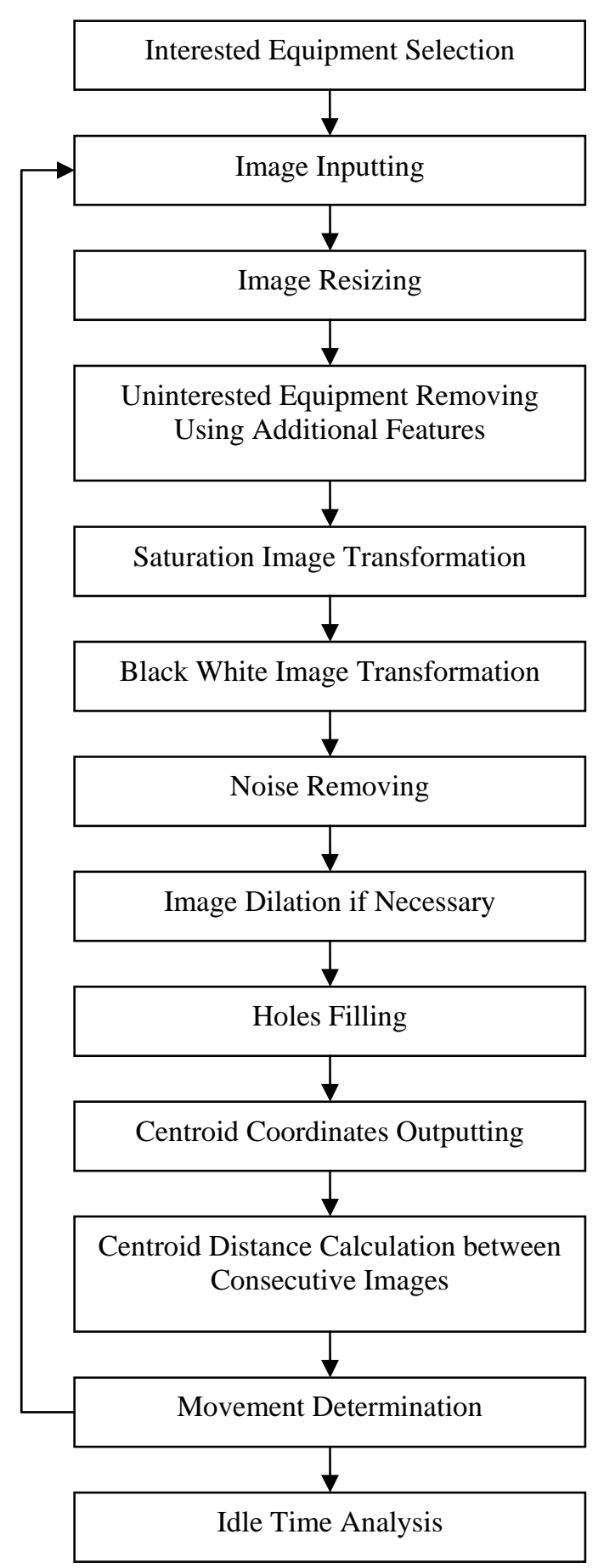

Fig.15 Flow Chart 